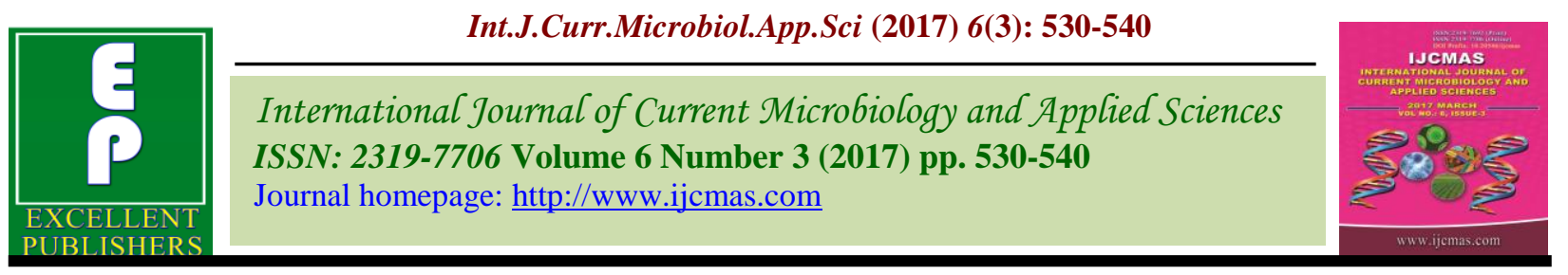

Original Research Article

https://doi.org/10.20546/ijcmas.2017.603.062

\title{
Green Synthesis and Characterization of Silver Nanoparticles from Gymnema sylvestre Leaf Extract: Study of Antimicrobial Activities
}

\author{
N. Supraja ${ }^{1}$, B. Avinash ${ }^{2}$ and T.N.V.K.V Prasad ${ }^{1}{ }^{*}$ \\ ${ }^{1}$ Acharya N. G. Ranga Agricultural University, Regional Agricultural Research Station, \\ Tirupati-517502, A.P. India \\ ${ }^{2}$ College of veterinary science Tirupati, Sri Venkateswara Veterinary University, \\ Tirupati, India \\ *Corresponding author
}

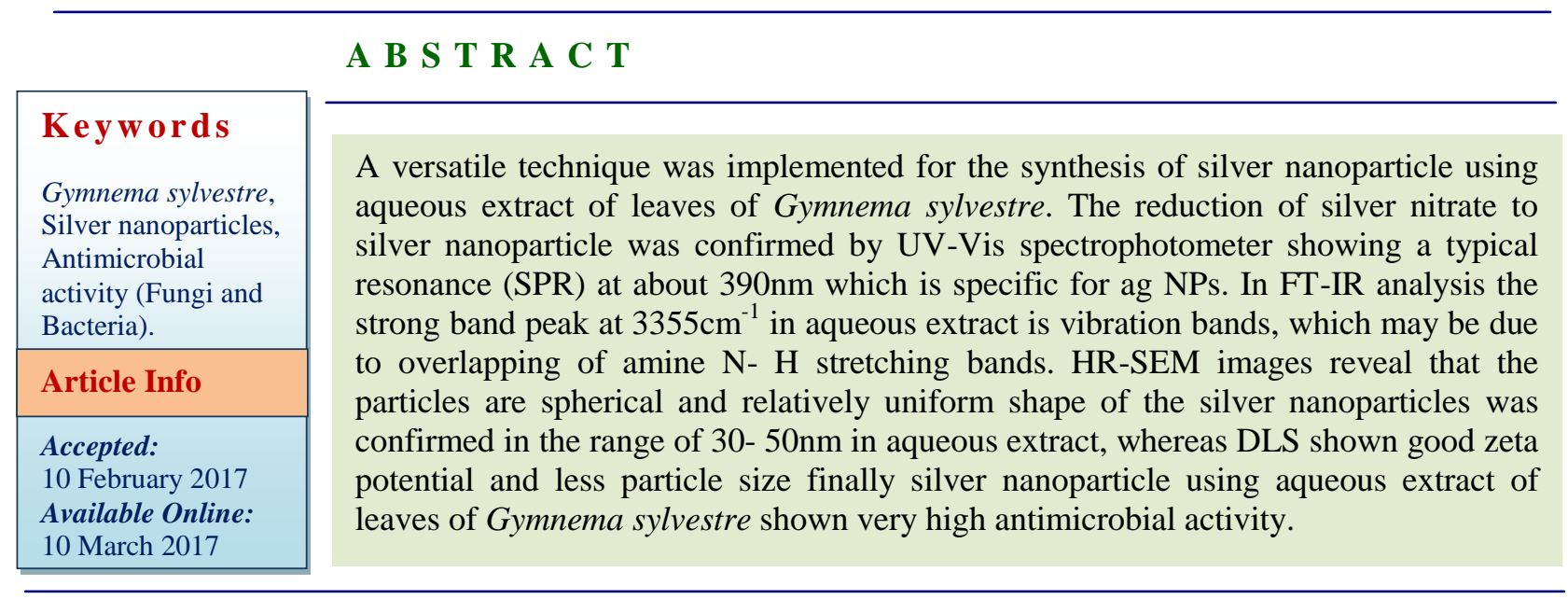

\section{Introduction}

Uncontrolled growth and spread of abnormal cells lead to cancer and finally results in death. Ethno-pharmacological process on the synthesis of nanoparticles is an amazing technology beneath construction symbiosis between nanoscience and medical sciences. In this regard, the idea of functionalizing gold nanoparticles for antidiabetic nanomaterial, by synthesizing pharmacologically key plant materials often been considered. Advances beneath nanotechnology have identified possible candidates for biological and biomedical programs on pharmaceutics, for novel diagnostics and medical agents. The nanoparticle drug delivery system has the advantages of accumulating large amounts of therapeutic drugs in the tumor tissues through the passive and active targeting approach (Mateos et al., 2013). Colon adenocarcinoma is the most common type of gastrointestinal cancer, and the National Institute of cancer has reported about 140,000 cases each year in the United States. This type of cancer begins in the inner layers of glandular structures of the colon cells and spreads into the wall of the colon and potentially into the lymphatic system and other organs (Ebrahimnejad et al., 2010). Gymnema sylvestre (GS), a plant used in the Indian Ayurvedic medicine for treating diabetes mellitus, has been known from antiquity also to have an anti saccharin taste effect. Many researchers have reported that 
the leaves of $G$. sylvestre lowers blood sugar, stimulates the heart, uterus, and circulatory systems, and exhibit anti sweet and hepatoprotective activities (El Shafey et al., 2013). Gymnema sylvestre has been used in the treatment of diabetes since ages in folk, ayurvedic and homeopathic systems of medicine. In addition, it also possesses antimicrobial, antitumor, obesity, anti Inflammatory and Anti-hyperglycemic Activity. Also in our previous studies we have reported that the bioactive compounds present in Gymnema sylvestre possess the anti-stress, anti-allergic, and antiulcer activity. Administration of $G$. sylvestre extract to diabetic rats increased superoxide dismutase activity and decreased lipid peroxide by directly scavenging the reactive oxygen species, due to the presence of various antioxidant compounds, or by increasing the synthesis of antioxidant molecules (albumin and uric acid) (Ramkumar et al., 2011). Presently, numerous methods namely physical, chemical substance, natural, as well as crossbreed techniques can be found in order to synthesize various kinds of precious metal Nanoparticles such as silver, gold, silicon, zinc and platinum nanoparticles. Even though actual physical as well as chemical substance techniques tend to be more wellknown as well as popular with regard to activity associated with nanoparticles, the actual associated environment degree of toxicity as well as non biodegradable character from the items restricted their own programs (Arun et al., 2014; Jayaseelan et al., 2013).

The green synthesized, characterized and biofunctionalized silver Nanoparticles from Gymnema sylvestre were tested for in vitro anticancer activity against human colon adenocarcinoma cells. Our present findings clearly demonstrated that it is indeed possible to have a much greener way to synthesize AgNPs without compromising their medicinal properties and thus plant extracts may prove to be a good alternative to obtain AgNPs with improved antibacterial properties.

\section{Materials and Methods}

\section{Materials}

Silver nitrate ( $>99 \%$ pure) was purchased from Sigma Aldrich, India. Potato dextrose broth, Potato dextrose agar, Nutrient broth, Nutrient agar plate, was supplied by $\mathrm{Hi}$ media, India.

\section{Sample collection and preparation} (Gymnema sylvestre, Leaves)

The leaves (Gymnema sylvestre) were collected from the S.V. Veterinary University, Tirupathi (Fig. 1), India and were brought to the nanotechnology laboratory and washed with distilled water several times to remove the impurities. The clean leaves were dried at room temperature in the shade for a week and powdered using a mortar and pestle.

Preparation of Gymnema sylvestre (leaf) extract

Dried powdered Gymnema sylvestre (5g) was mixed with $100 \mathrm{ml}$ distilled water then the solution was kept for continues heating at $80^{\circ} \mathrm{C}$ for 1 hour at room temperature with frequent shaking. After that the extract were filtered by using Whatmann No1 filter paper. The extract was collected and stored at $4{ }^{\circ} \mathrm{C}$ for further use.

Synthesis of silver nanoparticles from Gymnema sylvestre

$10 \mathrm{ml}$ of the aqueous extract of Gymnema sylvestre was added into $90 \mathrm{ml}$ of aqueous solution of $1 \mathrm{mM}$ Silver nitrate. The mixture was exposed to a range of controlled temperatures for $24 \mathrm{~h}$. Appearance of brown 
color in solution indicated the formation of AgNPs. The solution was then kept in dark for further analysis collected and stored at $4^{\circ} \mathrm{C}$ for further use.

\section{Collection of microbes (Bacteria and fungi)}

The microbes (Bacteria and fungi) samples were collected from Nanotechnology laboratory, Regional Agricultural Research Station, Tirupathi, (Chittoor District) Andhra Pradesh, India. These samples were stored in an ice box and transported to the laboratory for microbiological characterization. Through serial dilution pour plate technique, fungal $\mathrm{sp}$. was isolated using potato dextrose agar (PDA) medium, and Gram negative and Gram-positive bacteria were isolated from nutrient agar medium. Further, it is maintained in potato dextrose agar slants (fungi) and nutrient agar slants (bacteria) for onward analysis.

\section{Antibacterial activity of Gymnema sylvestre produced AgNPs}

The antibacterial activity of AgNPs was evaluated against the following pathogenic strains E. coli, Pseudomonas Fluorescence, Staphylococcus aureus and Bacillus subtilis. These cultures were grown on appropriate medium at $37^{\circ} \mathrm{C}$ for overnight incubation and maintained at $4^{\circ} \mathrm{C}$ in a refrigerator.

Disc diffusion method disc of 5Mm was made for nutrient agar medium and each disc was dipped at different concentration (170, 100, $50 \mathrm{ppm}$ ) efficiency of prepared AgNPs. The pure cultures of bacterial pathogens were subcultured on an appropriate medium. For comparison, plate of the same diameter with $5 \mathrm{Mm}$ Ampicillin (30mcg) was used. After incubation at $37^{\circ} \mathrm{C}$ for $24 \mathrm{~h}$ the zones of bacterial inhibition were measured. The assays were performed triplicate.
Antifungal activity of Gymnema sylvestre produced AgNPs

The antifungal activity of AgNPs was evaluated against the following pathogenic strains Aspergilus niger, Aspergilus flavus, Schelorosium rolfsii, Rhizopus oligosporus, and these cultures were grown on appropriate medium at $37^{\circ} \mathrm{C}$ for overnight incubation and maintained at $4^{\circ} \mathrm{C}$ in a refrigerator. Disc diffusion method disc of $5 \mathrm{Mm}$ was made on nutrient agar medium and each disc was dipped at different concentration (170, 100, $50 \mathrm{ppm}$ ) efficiency of prepared AgNPs. The pure cultures of fungal pathogens were subcultured on an appropriate medium. Discs of $5 \mathrm{~mm}$ diameter were made on potato dextrose agar medium. Each strain was swabbed uniformly onto the individual plate. For comparison, plate of the same diameter with $5 \mathrm{Mm}$ ketoconazole $(30 \mathrm{mcg})$ was used. After incubation at $37^{\circ} \mathrm{C}$ for $24 \mathrm{~h}$ the zones of fungal inhibition were measured. The assays were performed triplicate.

\section{Characterization of Ag nanoparticles}

\section{UV - Visible spectrum for synthesized nanoparticles}

The nanoparticles were monitored by UVvisible spectrum at various time intervals. The UV - Visible spectra of this solution was recorded in spectra 50 ANALYTIKJENA Spectrophotometer, from 250 to $400 \mathrm{~nm}$.

\section{FTIR analysis for synthesized nanoparticles}

The nanoparticles were harvested and characterized by FTIR. The FTIR spectrum was taken in the mid IR region of 400-4000 $\mathrm{cm}^{-1}$. The spectrum was recorded using ATR (attenuated total reflectance) technique. The sample was directly placed in the $\mathrm{KBr}$ crystal 
and the spectrum was recorded in the transmittance mode.

\section{X-ray diffraction analysis for synthesized nanoparticles}

The nanoparticles were harvested and characterized by XRD and TEM. The XRD pattern was recorded using computer controlled XRD-system, JEOL, and Model: JPX-8030 with $\mathrm{CuK}$ radiation $(\mathrm{Ni}$ filtered $=$ $\left.13418 \mathrm{~A}^{\circ}\right)$ at the range of $40 \mathrm{kV}, 20 \mathrm{~A}$. The 'peak search' and 'search match' program built in software (syn master 7935) was used to identify the peak table and ultimately for the identification of XRD peak.

\section{Particle size and zeta potential analyzer for synthesized nanoparticles}

The aqueous suspension of the synthesized nanoparticles was filtered through a $0.22 \mu \mathrm{m}$ syringe driven filter unit and the size of the distributed nanoparticles were measured by using the principle of Dynamic Light Scattering (DLS) technique made in a Nanopartica (HORIBA, SZ-100) compact scattering spectrometer.

\section{High resolution scanning electron microscope}

The structural morphological characteristics of the bacterial sample were observed under scanning electron microscope (HR-SEM) Hitachi's SU6600 at magnification ranging from $10 \mathrm{X}$ to $600,000 \mathrm{X}$ operated at accelerating voltage of $30 \mathrm{kv}$.

\section{Results and Discussion}

\section{UV-Visible spectral analysis}

It is well- known that silver nanoparticles exhibit brown color, which arises due to excitation of surface Plasmon vibrations of the silver nanoparticles. After addition of $1 \mathrm{mM}$ silver nitrate solution to the aqueous extract, the colour of the composition has been changed to dark brown colour. The maximum absorbance peak is observed at $390 \mathrm{~nm}$ for Gymnema sylvestre (Fig. 2). The overall observations suggest that the bio reduction of (silver ions) $\mathrm{Ag}^{+}$to $\mathrm{Ag}^{(0)}$ was confirmed by UV-Visible spectroscopy

\section{FT-IR analysis}

FT-IR spectrum of the biosynthesized silver nanoparticles using Gymnema sylvestre. (Fig. 3 ) shows the absorption peaks at 3355, 2989, 2885, 2822, 2104, 1772, 1637, 1406 and $1049 \mathrm{~cm}^{-1}$. The peak at $3355 \mathrm{~cm}^{-1}$ reveals the presence of $\mathrm{N}-\mathrm{H}$ stretching vibration, indicating the primary and secondary amines group of proteins, $2989 \mathrm{~cm}^{-1}$ reveals the presence of $\mathrm{C}-\mathrm{H}$ stretching vibration, indicating the presence of alkenes, $2885 \mathrm{~cm}^{-1}$ reveals the presence of $\mathrm{C}-\mathrm{H}$ stretching vibration, indicating the presence of alkanes, $2822 \mathrm{~cm}^{-1}$ reveals the presence of $\mathrm{C}-\mathrm{H}$ stretching vibration, indicating the presence of aldehydes, $2104 \mathrm{~cm}^{-1}$ reveals the presence of $-\mathrm{C} \equiv \mathrm{C}$ - stretching vibration, indicating the presence of alkynes, $1772 \mathrm{~cm}^{-1}$ reveals the presence of $\mathrm{C}=\mathrm{O}$ stretching vibration, indicating the presence of carbonyls in proteins, $1637 \mathrm{~cm}^{-1}$ reveals the presence of $\mathrm{N}$ $\mathrm{H}$ bend stretching vibration, indicating the presence of primary amines, $1406 \mathrm{~cm}^{-1}$ reveals the presence of $\mathrm{C}-\mathrm{C}$ stretching vibration, indicating the presence of aromatics, $1049 \mathrm{~cm}^{-}$ 1 reveals the presence of $\mathrm{C}-\mathrm{C}$ stretching vibration, indicating the presence of aliphatic amines respectively indicating the involvement of proteins in reduction and stabilization of silver ions, The position of these bands were comparable to phenols, flavonoids and tannins. Thus, we can confirm that the nano capping of the Gymnema sylvestre extract is responsible for the reduction and subsequent stabilization of the 
AgNPs. Gymnema sylvestre extract were coated by phyto compounds and secondary metabolites such as saponins, terpenoids and gymnemagenin derivative of gymnemic acid containing the functional groups of amines, aldehydes, carboxylic acids and alcohols.

\section{XRD analysis}

XRD analysis of AgNPs shows several size dependent features leading to regular peak position, height and width. XRD was mainly carried out to study the crystalline nature of the green synthesized Gymnema sylvestre silver nanoparticles. The diffraction intensities were recorded from $10^{\circ}-80^{\circ}$ at $2 \theta$ angles (Fig. 4). Five different and important characteristic peaks were observed at the $2 \theta$ of $38.6^{\circ}, 45.8^{\circ}, 58.7^{\circ}, 64.8^{\circ}$ and $78.6^{\circ}$ that correspond to (111), (200), (220), (311) and (222) planes indicating that are the SNPs are highly crystalline respectively.

Table.1 In vitro antibacterial studies against bacteria using Gymnema sylvestre extract mediated silver nanoparticles as inhibitors

\begin{tabular}{|c|c|c|c|c|c|}
\hline \multirow[t]{2}{*}{ S.No } & \multirow[t]{2}{*}{ Bacteria } & \multicolumn{4}{|c|}{ Gymnema sylvestre aqueous extract mediated synthesis of silver nanoparticles } \\
\hline & & 170ppm $\pm 1.4 \mathrm{ppm}$ & 100ppm $\pm 1.1 \mathrm{ppm}$ & 50ppm $\pm 0.8 p p m$ & Tetracycline 30mcg \\
\hline 1. & Escherichia Coli & $3.7 \pm 0.6$ & $2.7 \pm 0.3$ & $2.3 \pm 0.5$ & $0.3 \pm 0.02$ \\
\hline 2. & $\begin{array}{l}\text { Staphylococcus } \\
\text { aureus }\end{array}$ & $3.0 \pm 0.5$ & $2.7 \pm 0.5$ & $1.9 \pm 0.02$ & $0.2 \pm 0.03$ \\
\hline 3. & $\begin{array}{l}\text { Pseudomonas } \\
\text { fluorescence }\end{array}$ & $3.4 \pm 0.4$ & $3.0 \pm 0.4$ & $1.2 \pm 0.08$ & $0.2 \pm 0.04$ \\
\hline 4. & Bacillus subtilis & $3.7 \pm 0.6$ & $2.4 \pm 0.3$ & $1.8 \pm 0.05$ & $0.3 \pm 0.02$ \\
\hline
\end{tabular}

*The presented data are the mean $(\mathrm{n}=3) \pm$ standard error of three replicates

Table.2 In vitro antifungal studies against fungi using Gymnema sylvestre extract mediated silver nanoparticles as inhibitors

\begin{tabular}{|c|c|c|c|c|c|}
\hline \multirow[t]{2}{*}{ S.No } & \multirow[t]{2}{*}{ Fungi } & \multicolumn{4}{|c|}{ Gymnema sylvestre aqueous extract mediated synthesis of silver nanoparticles } \\
\hline & & 170ppm $\pm 1.4 p p m$ & 100ppm $\pm 1.1 p p m$ & 50ppm $\pm 0.8 p p m$ & Ketoconazole 30mcg \\
\hline 1. & Aspergillus flavus & $1.3 \pm 0.05$ & $0.9 \pm 0.02$ & $0.6 \pm 0.01$ & $0.8 \pm 0.02$ \\
\hline 2. & Sclerotium rolfsii & $1.3 \pm 0.03$ & $0.8 \pm 0.04$ & $0.5 \pm 0.02$ & $1.6 \pm 0.06$ \\
\hline 3. & Aspergillus niger & $1.9 \pm 0.06$ & $1.2 \pm 0.03$ & $1.0 \pm 0.02$ & $0.9 \pm 0.03$ \\
\hline 4. & Rhizopus oligosporus & $0.8 \pm 0.03$ & $0.6 \pm 0.01$ & $0.3 \pm 0.03$ & $1.0 \pm 0.05$ \\
\hline
\end{tabular}

$*$ The presented data are the mean $(\mathrm{n}=3) \pm$ standard error of three replicates

Fig.1 Gymnema sylvestre

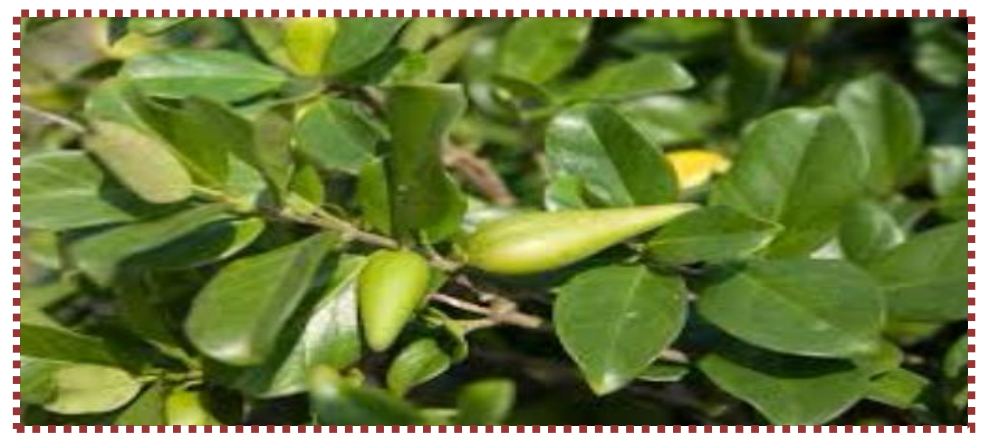


Fig.2 UV/Visible absorption spectrum of synthesized silver nanoparticles from Gymnema sylvestre

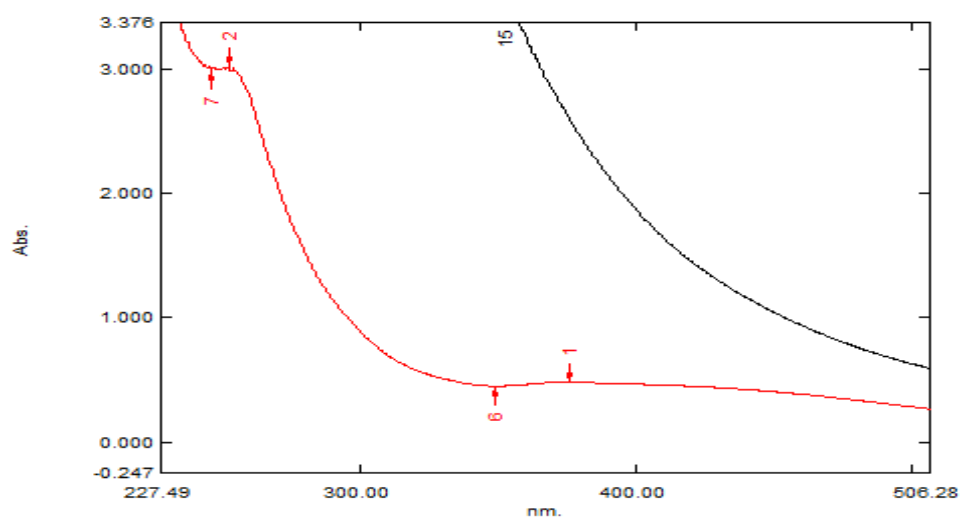

Fig.3 FT-IR spectrum of synthesized silver nanoparticles from Gymnema sylvestre

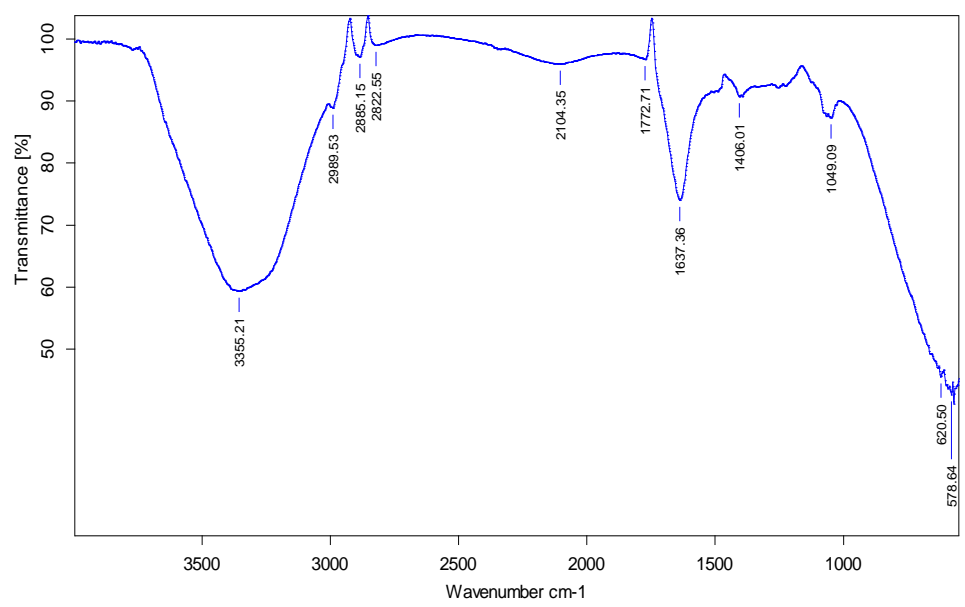

Fig.4 XRD analyses of synthesized silver nanoparticles from Gymnema sylvestre

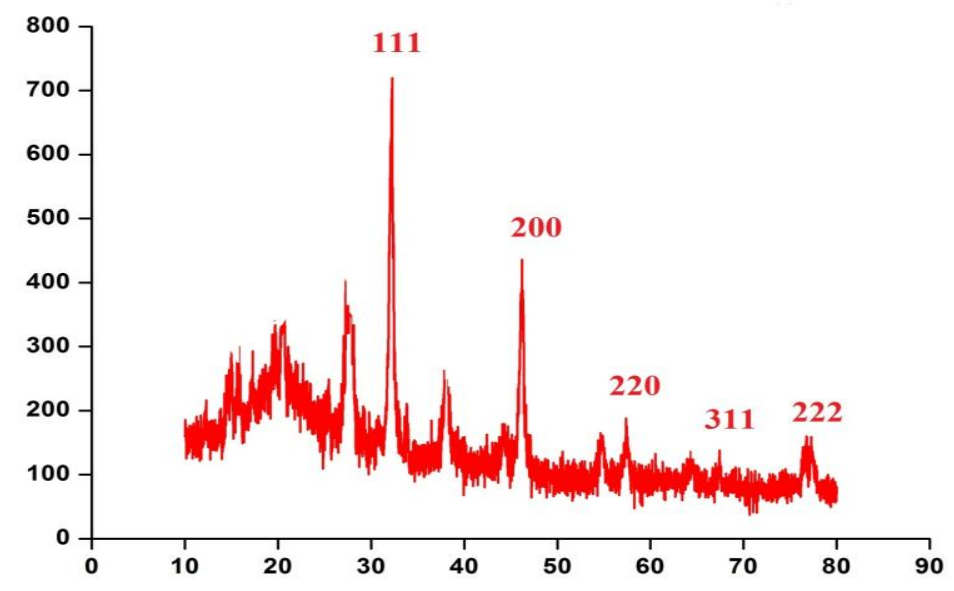


Fig.5 (a) Particle size; (b) Zeta potential (DLS analysis of synthesized silver nanoparticles from Gymnema sylvestre)
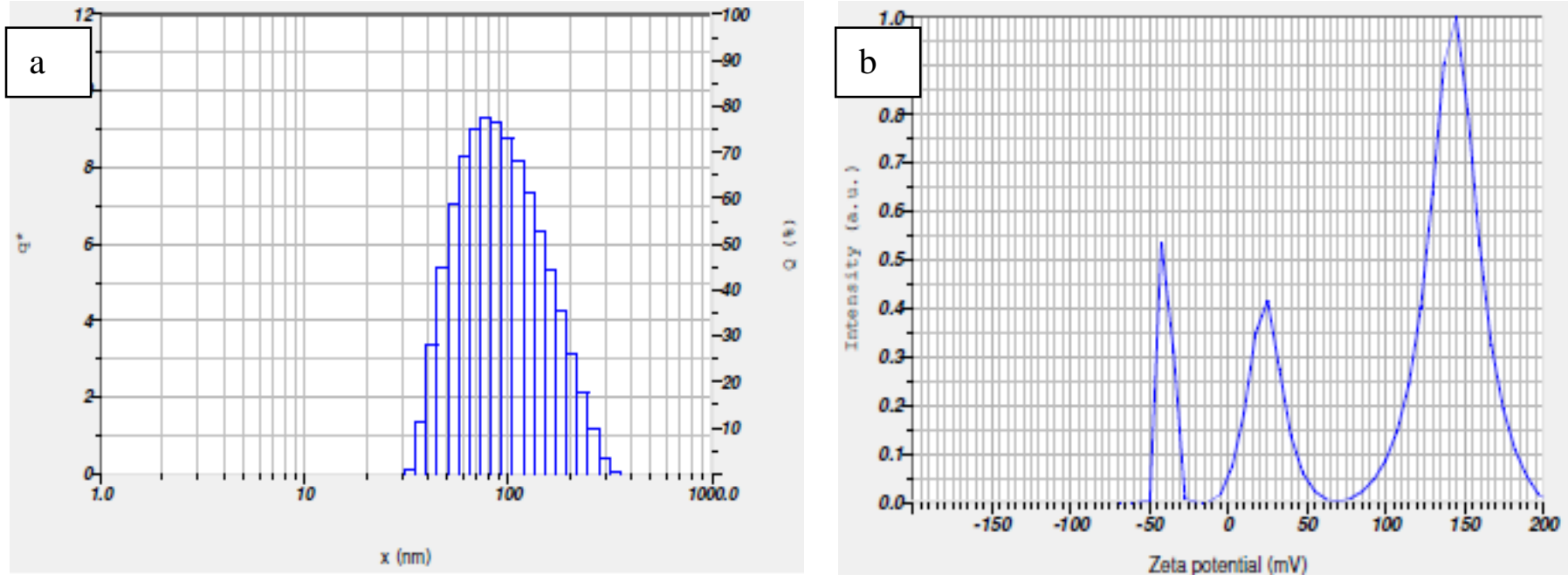

Calculation Results

\begin{tabular}{|c|c|c|c|c|}
\hline Pookinio. & S.PAas a Fatio & Noan & 50 & Modo \\
\hline 1 & TOOO & $72 K 9 \min$ & 34.7 nim & $126.5 \mathrm{~nm}$ \\
\hline 2 & - & $--\mathrm{nm}$ & $--\operatorname{mm}$ & $-\operatorname{mm}$ \\
\hline 3 & $=$ & $=\mathrm{nm}$ & $--\mathrm{nm}$ & $-\mathrm{nm}$ \\
\hline Total & 1.00 & $1279 \mathrm{~nm}$ & $307 \mathrm{~nm}$ & $125.5 \mathrm{~nm}$ \\
\hline \multicolumn{5}{|c|}{ Cumulant Operations } \\
\hline \multicolumn{3}{|c|}{$\begin{array}{l}\text { Z-Averacie } \\
\text { PI }\end{array}$} & : & $86.9 \mathrm{~mm}$ \\
\hline \multicolumn{5}{|c|}{ Molecular weight measurement } \\
\hline \multicolumn{4}{|c|}{ Molecular weight } & - \\
\hline \multicolumn{4}{|c|}{ Mark-Houmink-Sakurada parameters } & - \\
\hline
\end{tabular}

Calculation Results

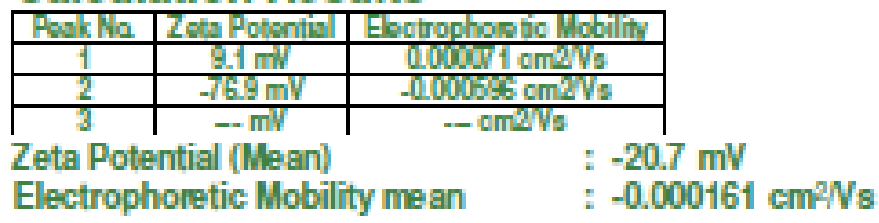

Fig.6 SEM analyses of synthesized silver nanoparticles from Gymnema sylvestre
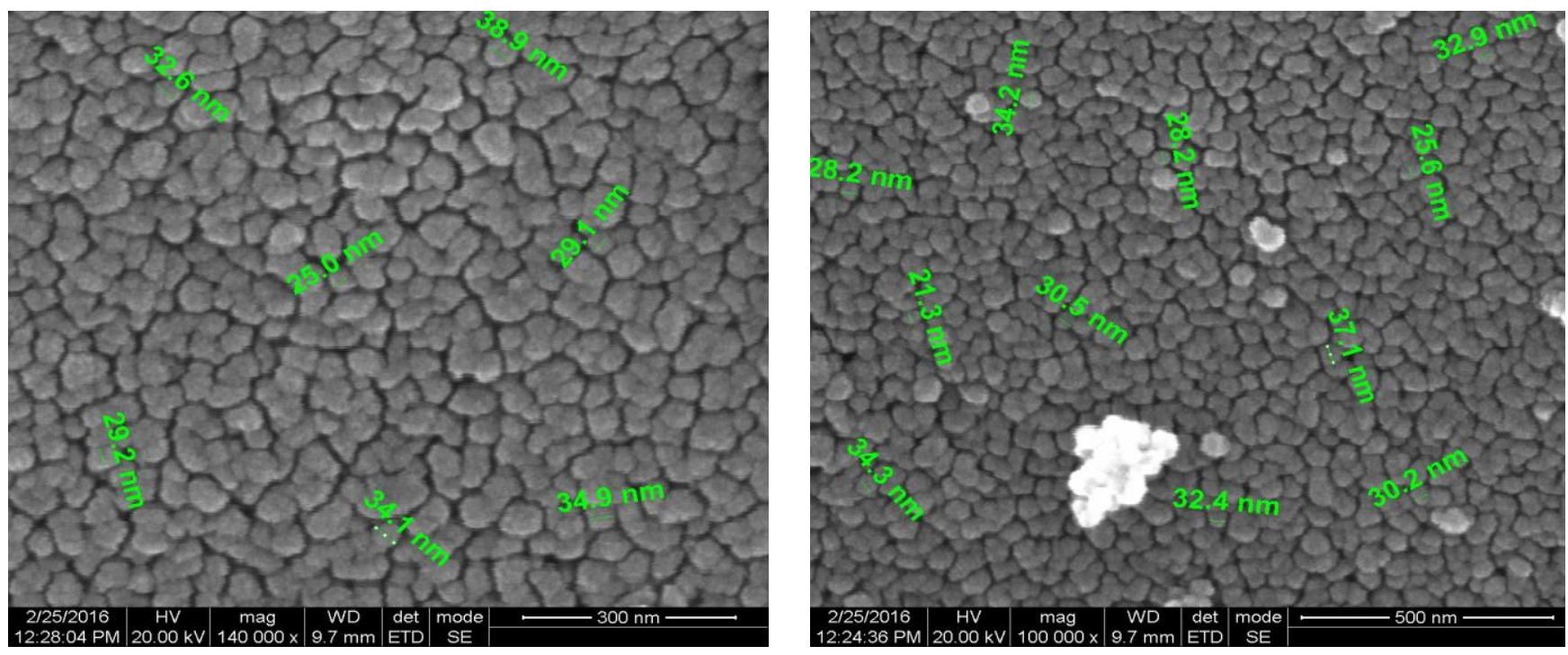
Fig.7a Anti bacterial activity of synthesized silver nanoparticles from Gymnema sylvestre

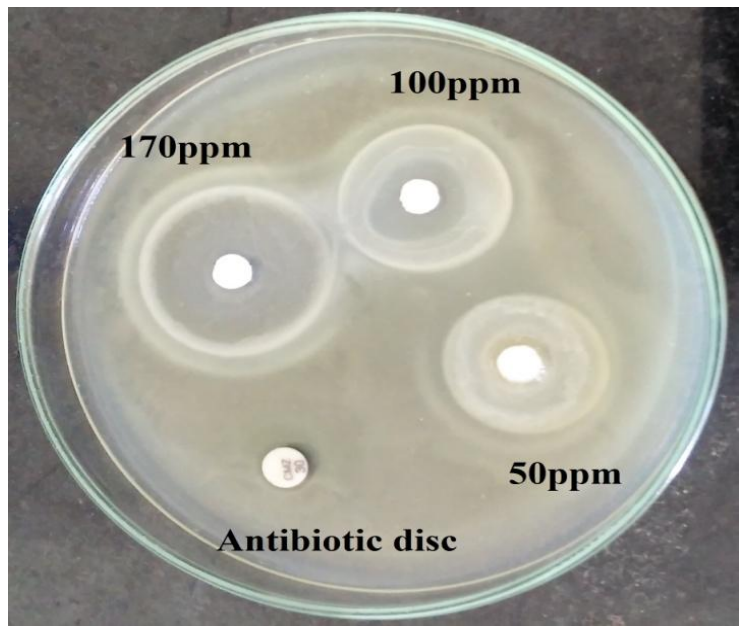

Escherichia coli

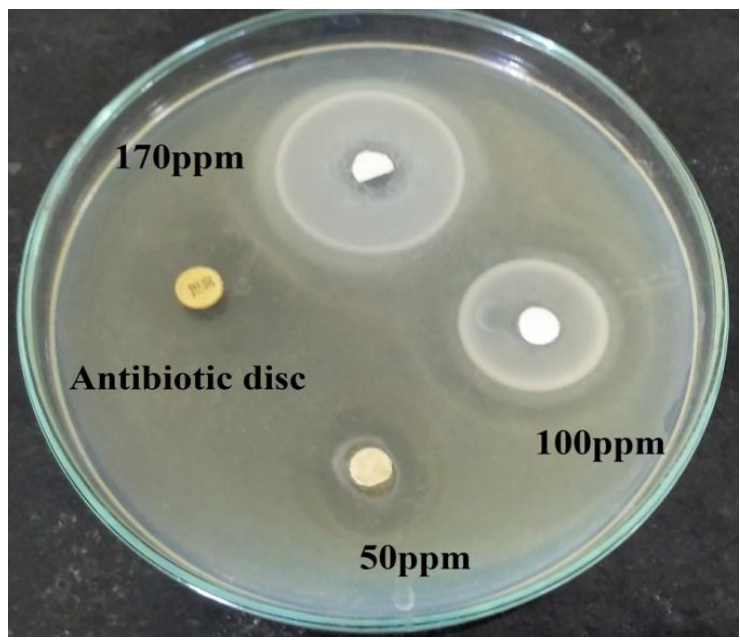

Pseudomonas fluorescence

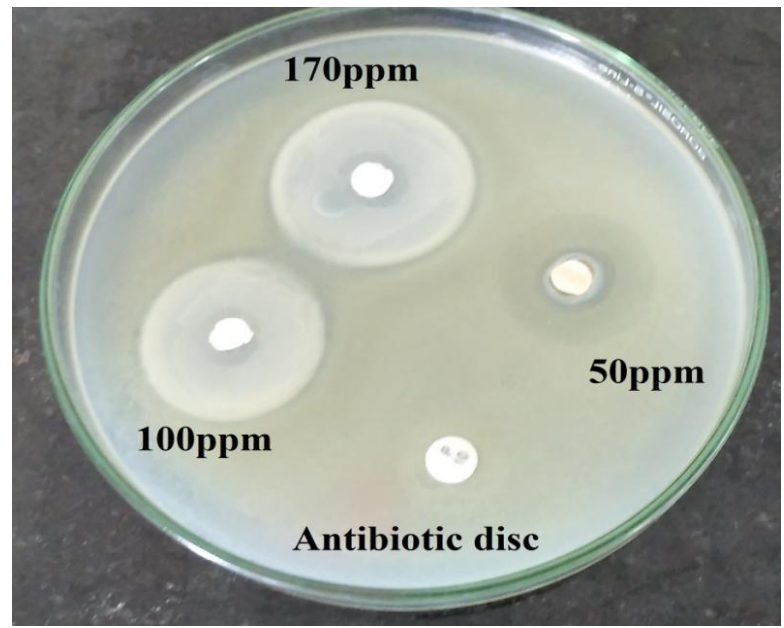

Staphylococcus aureus

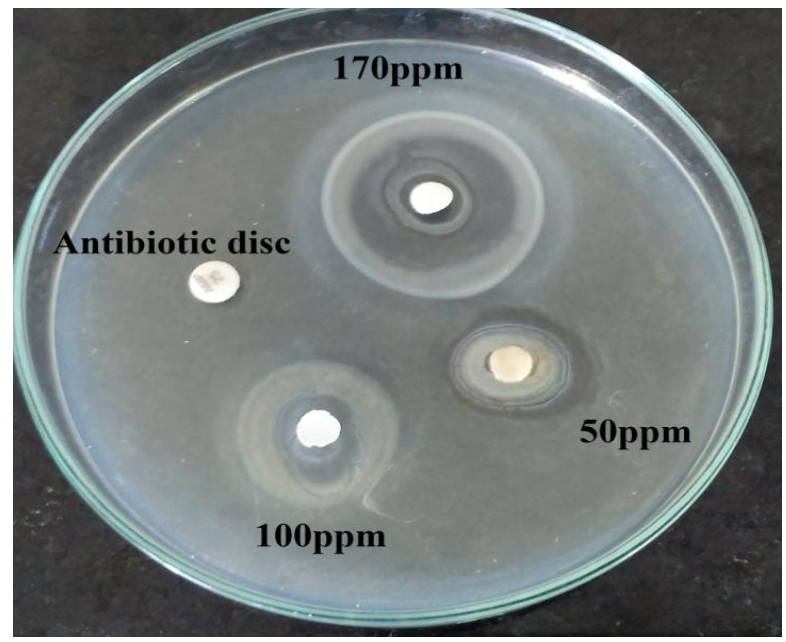

Bacillus subtilis

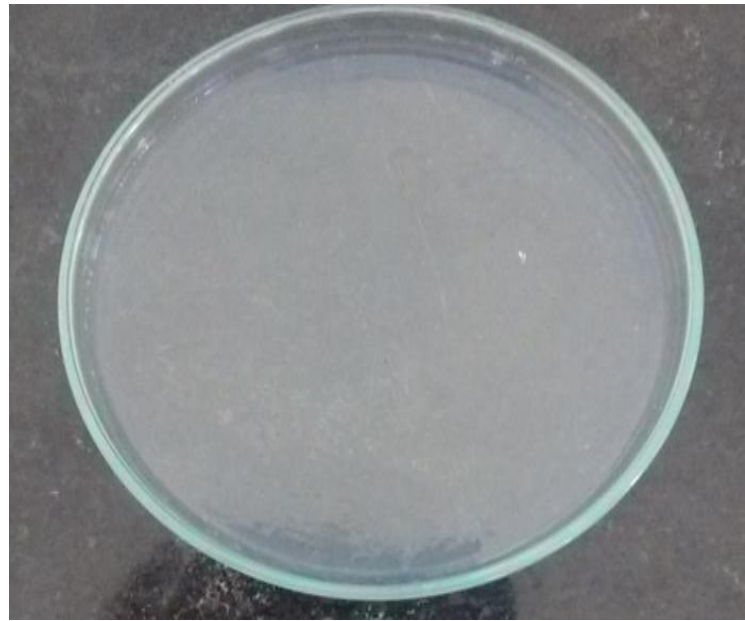

Control 
Fig.7b Anti fungal activity of synthesized silver nanoparticles from Gymnema sylvestre

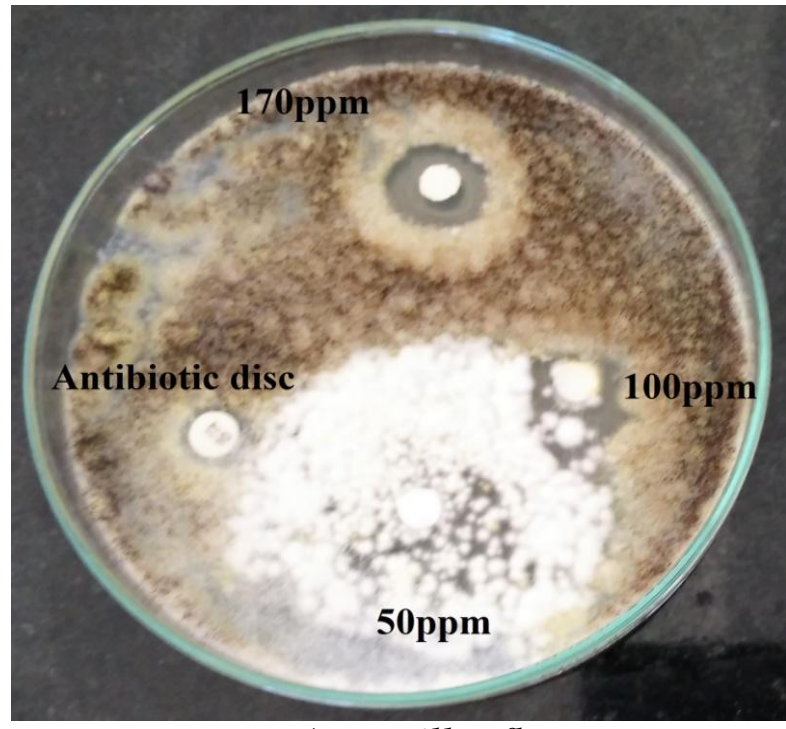

Aspergillus flavus

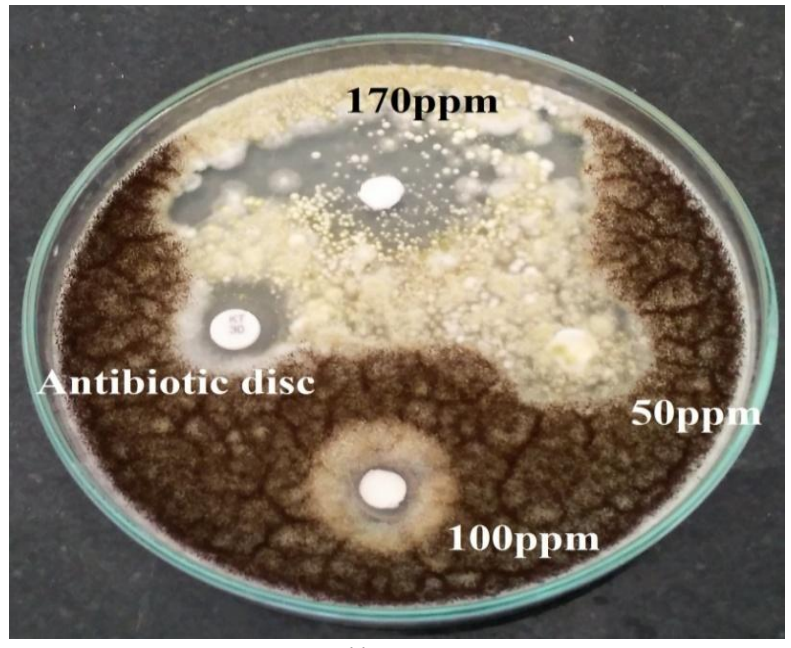

Aspergillus niger

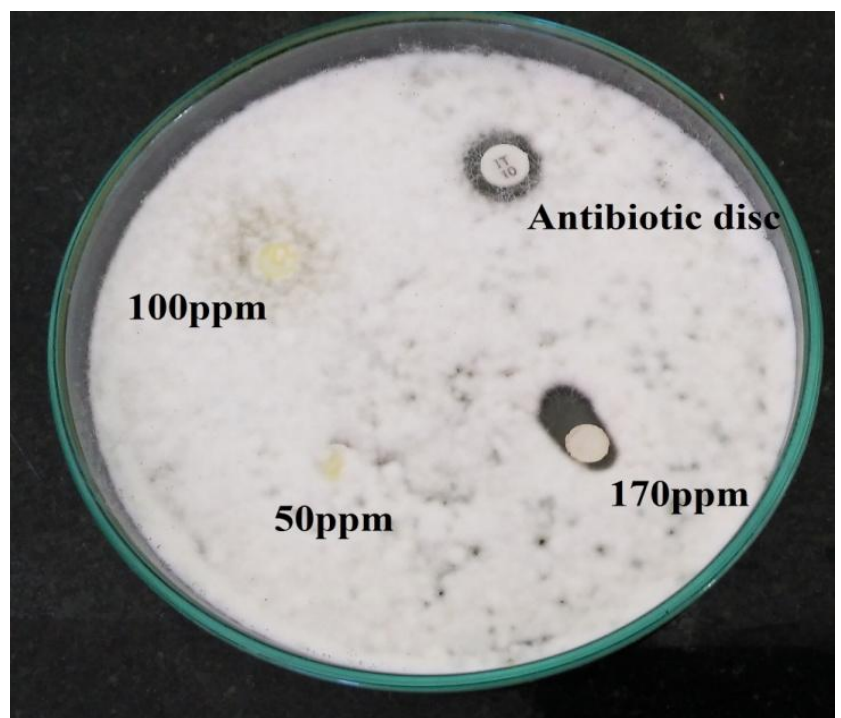

Sclerotium rolfsii

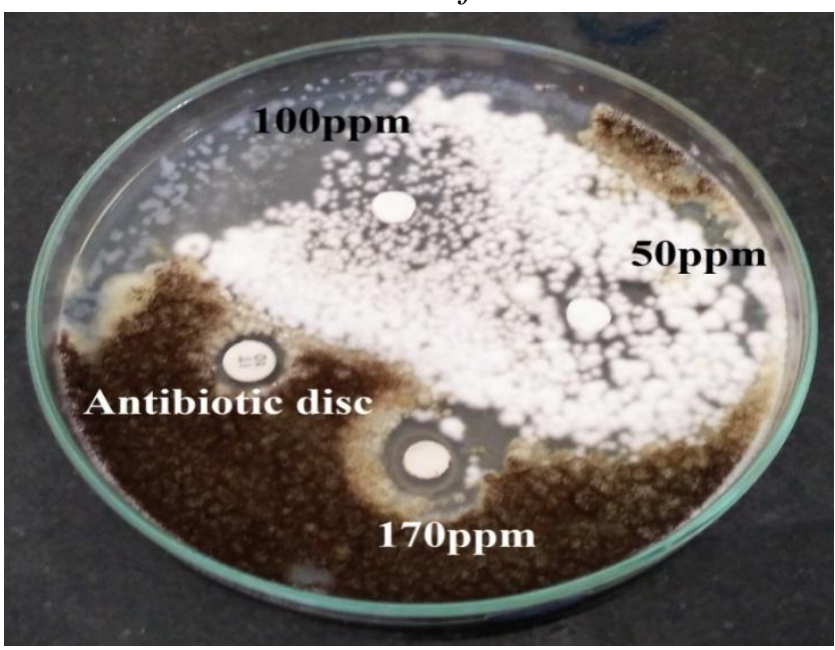

Rhizopus oligosporus

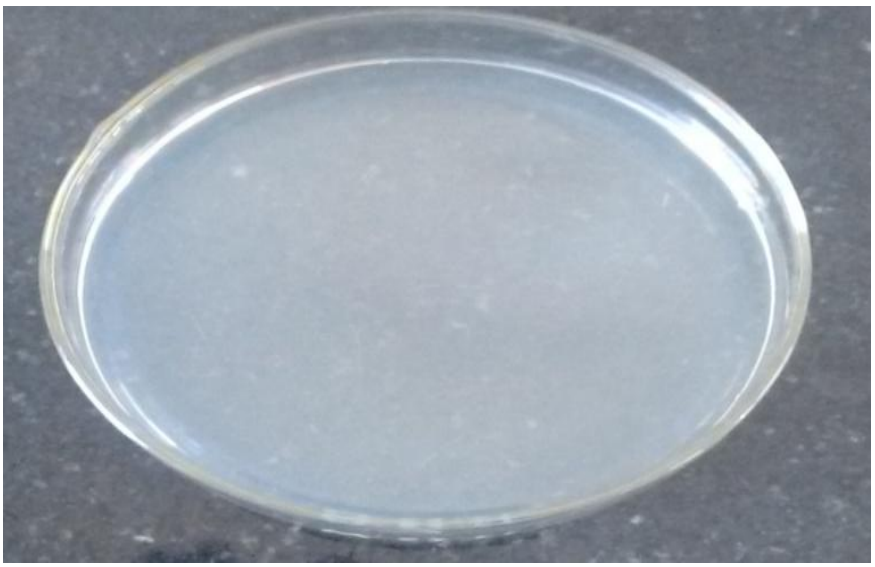

Control 


\section{Dynamic light scattering analysis}

The particle size distribution spectra for the silver nanoparticles were recorded as diameter $(\mathrm{nm})$ verses frequency $(\% / \mathrm{nm})$ spectra with diameter (nm) on $\mathrm{x}$-axis and frequency $(\% / \mathrm{nm})$ on $\mathrm{y}$-axis. The zeta potential spectra for the silver nanoparticles were recorded zeta potential verses intensity spectra with zeta potential $(\mathrm{mV})$ on $\mathrm{x}$-axis and intensity (a.u) on y-axis. Dynamic light scattering technique has been used to measure hydrodynamic diameter of the hydrosol (particle suspension). Gymnema sylvestre AgNPs was found to be $86.9 \mathrm{~nm}$ (Fig. 5a) the recorded value of zeta potential of the silver nanoparticles was $-20.7 \mathrm{mV}$ (Fig. 5b) which resulted in the agglomerated state of the formed AgNPs.

\section{HR-SEM analysis}

Gymnema sylvestre silver nanoparticles were characterized from the SEM micrograph, it is evident that AgNPs were spherical in shape and were poly-dispersed. The measured average size of AgNPs was $35 \mathrm{~nm}$ and the nanoparticles ranges from $25.0 \mathrm{~nm}$ to $37.1 \mathrm{~nm}$ occasional agglomeration of the AgNPs has been observed (Fig. 6). The more stable spherical shape and isotropic nanoparticles was formed by the action of large number of bimolecules ranged in the solution.

\section{Antimicrobial activity of Gymnema sylvestre extracts mediated silver nanoparticles}

It is well- known that silver nanoparticles exhibit brown color, arising due to excitation of surface Plasmon vibrations in the silver nanoparticles. Silver nanoparticles obtained from Gymnema sylvestre shown have very strong inhibitory action against fungal sp, Gram-positive and Gram-negative bacteria. These isolates were collected from nanotechnology laboratory, Acharya N G Ranga Agricultural University, Tirupathi. Three concentrations of NPs $(170,100$, $50 \mathrm{ppm}$ ) were prepared and were applied against an array of bacterial species viz., Escherichia coli, Staphylococcus aureus, Pseudomonas fluorescence and Bacillus subtilis, fungal species viz., Aspergillus flavus, Sclerotium rolfsii, Aspergillus niger and Rhizopus oligosporus. The higher concentration (170ppm) of AgNPs showed significant antimicrobial effect compared with other concentrations (100 and 50ppm).

The mechanism by which the nanoparticles are able to penetrate the bacteria is not understood completely, but studies suggest that when bacteria were treated with silver nanoparticles, changes took place in its membrane morphology that produced a significant increase in its permeability affecting proper transport through the plasma membrane (Auffan et al., 2009), leaving the bacterial cells incapable of properly regulating transport through the plasma membrane, resulting into cell death (Supraja et al., 2015). It is observed that silver nanoparticles have penetrated inside the bacteria and have caused damage by interacting with phosphorus- and sulfurcontaining compounds such as DNA (He et al., 2008). Moreover, Gymnema sylvestre AgNPs showed good antibacterial and antifungal activity (Fig. 7a and 7b) (Table 1 and 2). The findings in this study may lead to the development of AgNPs-based new antimicrobial systems for medical applications.

In conclusion, the silver nanoparticles were biologically synthesized from Gymnema sylvestre was economical, nontoxic and environmentally benign. Due to the reducing and capping nature of the bioactive phytocompounds, present in the aqueous extracts. This extracts is found to suitable for 
the green synthesis of silver nanoparticles with in 30minutes at ambient conditions. Color change occurs due to surface plasmon vibrations of the NPs which were confirmed by UV-Visible absorption spectroscopy. The presence of the functional group of the bioactive compounds was confirmed by FTIR and the particle size of NPs was determined by XRD, DLS. Bionanoparticles were characterized through various studies, in the HR-SEM analysis showed that the particles were spherical and the size of the particles ranges from $30 \mathrm{~nm}-60 \mathrm{~nm}$.

\section{References}

Arun, L.B., Arunachalam, A.M., Arunachalam, K.D., Annamalai, S.K., Amit Kumar, K. 2014. In vivo antiulcer, anti-stress, anti-allergic, and functional properties of Gymnemic Acid Isolated from Gymnema sylvestre $\mathrm{R}$ Br. BMC Complement Altern. Med., 14: 70

Auffan, M., Rose, J., Bottero, J.Y., Lowry, G.V., Jolivet, J.P., Wiesner, M.R. 2009. Towards a definition of inorganic nanoparticles from an environmental, health and safety perspective. Nat. Nanotechnol., 4: 634-641.

Ebrahimnejad, P., Dinarvand, R., Sajadi, A., Jaafari, M.R., Nomani, A.R., Azizi, E., et al. 2010. Preparation and in vitro evaluation of actively targetable nanoparticles for $\mathrm{SN}-38$ delivery against HT-29 cell lines. Nanomed., 6: 478-85.

El Shafey, A.M., El-Ezabi, M.M., Seliem, M.M.E., Ouda, H.H.M., Ibrahim, D.S.
2013. Effect of Gymnema sylvestre R.

$\mathrm{Br}$. leaves extract on certain physiological parameters of diabetic rats. J. King Saud Univ. Sci., 25: 135141.

He, Y., Wan, T.J.M., Tokunaga, T. 2008. Kinetic stability of hematite nanoparticles of the effect of particle sizes. J. Nanoparticle Res., 10: 321332.

Jayaseelan, C., Ramkumar, R., Rahuman, A.A., Perumal, P. 2013. Green synthesis of gold nanoparticles using seed aqueous extract of Abelmoschus esculentus and its antifungal activity. Ind. Crops Prod., 45: 423-429.

Mateos, R., Pereira-Caro, G., Bacon, J.R., Bongaerts, R., Sarriá, B., Bravo, L., et al. 2013. Anticancer Activity of Olive Oil Hydroxytyrosyl Acetate in Human Adenocarcinoma Caco-2 Cells. J. Agric. Food Chem., 61: 3264-3269.

Ramkumar, K.M., Vanitha, P., Uma, C., Suganya, N., Bhakkiyalakshmi, E., Sujatha, J. 2011. Antidiabetic activity of alcoholic stem extract of Gymnema montanum in streptozotocin-induced diabetic rats. Food Chem. Toxicol., 49: 3390-4.

Supraja, N., Prasad, T.N.V.K.V., Giridhar Krishna, T., David, E. 2015. Synthesis, Characterization and Evaluation of the antimicrobial efficacy of Boswellia ovalifoliolata stem bark extract mediated zinc oxide nanoparticles. Appl. Nanosci., DOI 10.1007/s13204015-0472-0

\section{How to cite this article:}

Supraja, N., Avinash, B. and Prasad, T.N.V.K.V. 2017. Green Synthesis and Characterization of Silver Nanoparticles from Gymnema sylvestre Leaf Extract: Study of Antimicrobial Activities. Int.J.Curr.Microbiol.App.Sci. 6(3): 530-540. doi: https://doi.org/10.20546/ijcmas.2017.603.062 